\title{
Brief
}

\section{Coronary-coronary bypass and pulmonary artery reconstruction related to Wegener granulomatosis in a 22-year-old woman}

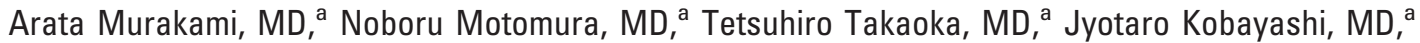 \\ Katsuhide Maeda, MD, ${ }^{\text {a }}$ Tetsushi Yamamoto, MD, ${ }^{\text {a }}$ Masahide Chikada, MD, ${ }^{\mathrm{b}}$ Toshiya Ootsuka, MD, \\ Yutaka Kotsuka, MD, and Shinichi Takamoto, MD, ${ }^{\text {a }}$ Tokyo, Japan
}

From the Department of Cardiothoracic Surgery, Faculty of Medicine, University of Tokyo, ${ }^{a}$ and the Department of Cardiovascular Surgery, National Children's Hospital, ${ }^{\mathrm{b}}$ Tokyo, Japan.

Received for publication May 21, 2002; accepted for publication July 3, 2002

Address for reprints: Arata Murakami, MD, Department of Cardiothoracic Surgery, Faculty of Medicine, University of Tokyo, 7-3-1, Hongou, Bunkyou-ku, Tokyo, 1138655, Japan (E-mail: MURAKAMITHO@h.u-tokyo.ac.jp).

J Thorac Cardiovasc Surg 2003;125:721-3

Copyright $(\odot) 2003$ by The American Association for Thoracic Surgery

0022-5223/2003 $\$ 30.00+0$

doi:10.1067/mtc.2003.14

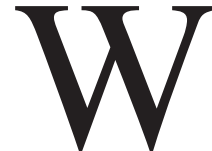

e report a case of left coronary artery ostial stenosis and bilateral pulmonary artery (PA) stenosis in a 22-year-old woman with Wegener granulomatosis (WG). Complicating periaortitis and obstruction of the internal thoracic arteries hampered conventional coronary artery bypass grafting and in situ PA reconstruction. Successful treatment was achieved by restoration of right ventricular outflow tract (RVOT) to bilateral PA continuities with reinforced polytetrafluoroethylene (PTFE) grafts and coronary-coronary bypass.

\section{Clinical Summary}

A 22-year-old woman was seen with chest pain. The diagnosis of WG was based on the eye, ear, and nose findings. Prednisolone had been started when the patient was 3 years old and continued for 4 years. A left ocular tumor was removed when she was 6 years old. A heart murmur was detected, and balloon pulmonary angioplasty was performed when she was 7 years old. Chest pain developed at 19 years of age. A $75 \%$ left coronary artery ostial stenosis (Figure 1, A) and bilateral branch PA stenoses were diagnosed when the patient was 21 years old and an operation was performed at another hospital. This operation consisted of two aorta-coronary bypass procedures with saphenous venous grafts and RVOT reconstruction with a Y-shaped woven Dacron polyester fabric graft (Hemashield vascular graft; Boston Scientific Corporation, Natick, Mass). The PA confluence was lost during the procedure. The right branch of the Dacron polyester fabric graft crossed anterior of the aorta and connected to the right PA, and the left branch was connected to the left PA in end-to-end fashion. The operation resulted in occlusion of the venous grafts and stenosis of the branches (Figure 1, $B$ ). The right to left ventricular pressure ratio after the operation was calculated as 0.95 , and stress-induced myocardial ischemia necessitated reoperation. The patient was referred to us 1 year after that operation.

Computed tomography demonstrated thickening of the wall of the ascending aorta, with an internal diameter of $13 \mathrm{~mm}$. The spaces between the sternum and the RVOT and aorta seemed wide enough to be used as an approach for right PA reconstruction. Both internal thoracic arteries were obstructed, and the left radial artery was hypoplastic.

The erythrocyte sedimentation rate, C-reactive protein, coagulation study, and serum antineutrophilic cytoplasmic antibody values were within normal ranges. The result for von Willebrand factor showed $110 \%$ activity.

At reoperation, after groin cannulation, the Y-shaped Dacron polyester fabric graft, including both branches, was removed, and pliable distal native PA stumps were prepared at each side. The aorta was identified behind the right branch of the Dacron polyester fabric graft and was surrounded by a dense fibrous reaction. There was no room to clamp the ascending aorta.

Muscle resection of the RVOT was added to provide an appropriate diameter. A 12-mm reinforced PTFE graft was anastomosed to the right side distal PA stump in end-to-end fashion, and another graft was connected to the left side PA by the same manner. About 10 $\mathrm{mm}$ each of the proximal parts of these two grafts were connected to each other in side-to-side 

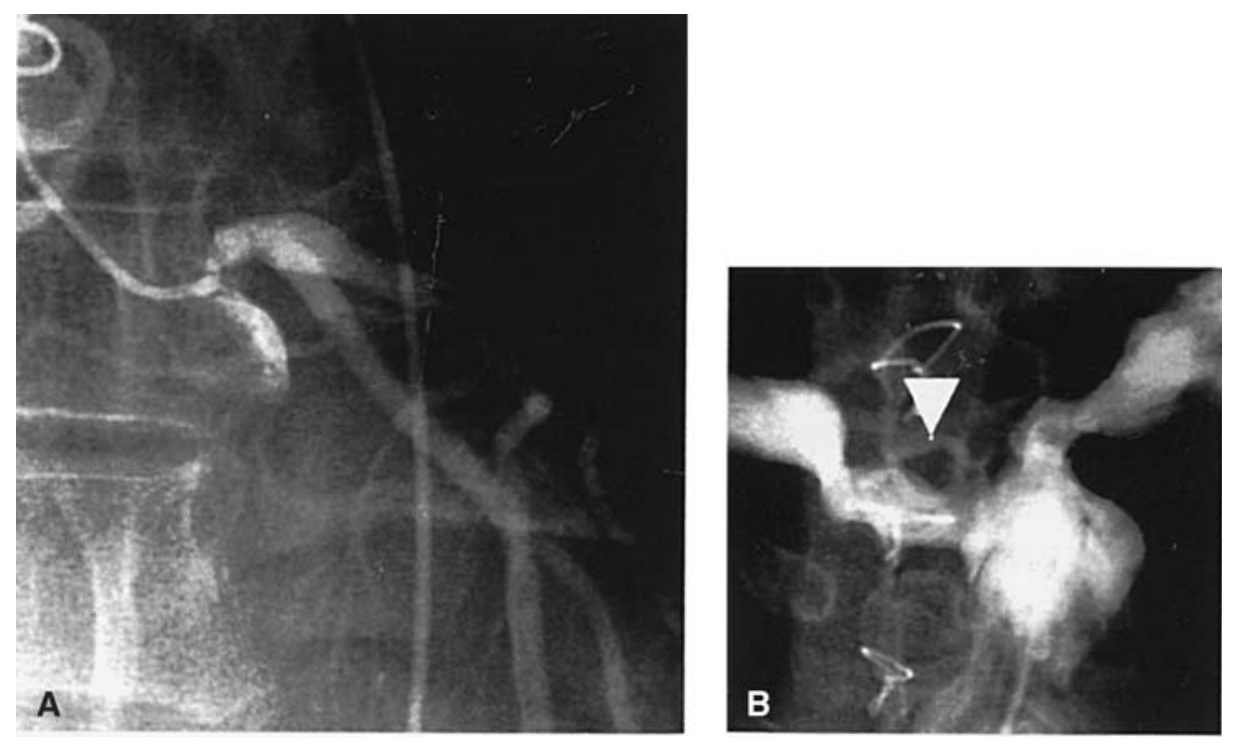

Figure 1. A, Coronary angiography demonstrates $75 \%$ stenosis of left coronary ostium. B, Both branches of Dacron polyester fabric graft show stenosis. Locator (white triangle) was placed on proximal anastomosis of saphenous venous graft.

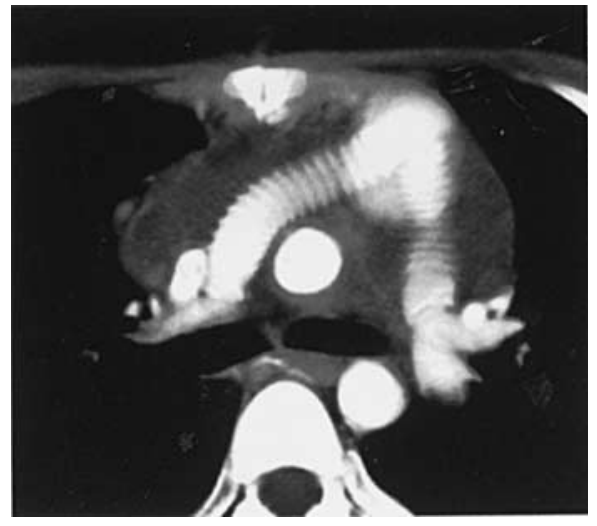

Figure 2. Reinforced PTFE grafts remained patent.

fashion to form a main PA portion and then sutured to the opening at the center of the woven Dacron polyester fabric patch newly placed on the RVOT.

Finally, coronary-coronary bypass was accomplished with a saphenous venous graft between the right coronary artery and the left coronary system. The entire procedure was completed with the heart beating under mild hypothermia. Intraoperative determination of the right to left ventricular pressure ratio yielded 0.4 .

Postoperative computed tomography demonstrated patency of the venous graft and PTFE grafts (Figure 2).

\section{Discussion}

WG, an antineutrophilic cytoplasmic antibody-associated systemic vasculitis, is clinically manifested by ocular, ear, nose, lung, and kidney involvement in most cases, and small to medium sized vasculitis is the underlying pathophysiologic basis of these clinical manifestations. ${ }^{1,2}$ In this case, the pathologic examination of the ocular tumor resected when the patient was 6 years old was not specific for WG, and the diagnosis was made on the basis of the clinical manifestations. The highly diseased aorta suggested inflammation in the past, and the periaortitis was presumed to be related to the ostial stenosis of the left coronary artery and the PA stenosis. On the other hand, combined with the occlusion of the bilateral internal thoracic arteries, the periaortitis hampered conventional coronary artery bypass and in situ PA reconstruction. These atypical manifestations of WG have not previously been reported in the literature.

With regard to the RVOT reconstruction, the reinforced PTFE grafts offered good patency even in the nonanatomic route. Use of a valved allograft ${ }^{3}$ would have resulted in stenosis of the branches, especially the right one, because of undue wide angulation from the main PA portion in the nonphysiologic routes.

With respect to coronary revascularization, the factors described previously warranted coronary-coronary bypass. ${ }^{4}$ Catheter intervention, including stent placement or dilation by a rotablator, for nonatherosclerotic ostial stenosis would have involved a high risk. Direct surgical enlargement of the left coronary ostium or Dacron polyester fabric graft replacement of the diseased aorta ${ }^{5}$ with the aid of deep hypothermic circulatory arrest did not seem promising in this case. A bypass graft to the left coronary system with a proximal implantation on an arch vessel would have been compressed by the bulky right side PTFE graft over the aorta. We abandoned the use of the right radial artery or the gastroepiploic artery as graft material in view of the risk of vasculitis. Although the quality of the saphenous vein seemed appropriate, long-term careful monitoring of its patency is required. 


\section{References}

1. Russell KA, Fass DN, Specks U. Antineutrophil cytoplasmic antibodies reacting with the pro form of proteinase 3 and disease activity in patients with Wegener's granulomatosis and microscopic polyangiitis. Arthritis Rheum. 2001;44:463-8.

2. Rasmussen N. Management of the ear, nose, and throat manifestations of Wegener's granulomatosis. Curr Opin Rheumatol. 2001;13:3-11.
3. Kreutzer C, Vive JD, Oppido G, Kreutzer J, Gauvreau K, Freed M, et al. Twenty-five-year experience with Rastelli repair for transposition of the great arteries. J Thorac Cardiovasc Surg. 2000;120:21123.

4. Nottin R, Grinda JM, Anidjar S, Folliguet T, Detroux M. Coronarycoronary bypass graft: an arterial conduit-sparing procedure. J Thorac Cardiovasc Surg. 1996;112;1123-30.

5. Ott DA, Cooley DA. The difficult proximal coronary anastomosis. Cardiovasc Dis Bull Tex Heart Inst. 1979;6:55-8.

\section{Acute graft failure caused by an intracoronary shunt in minimally invasive direct coronary artery bypass grafting}

Hironori Izutani, MD, PhD, and Inderjit S. Gill, $\operatorname{FRCS(C),~Cleveland,~Ohio~}$

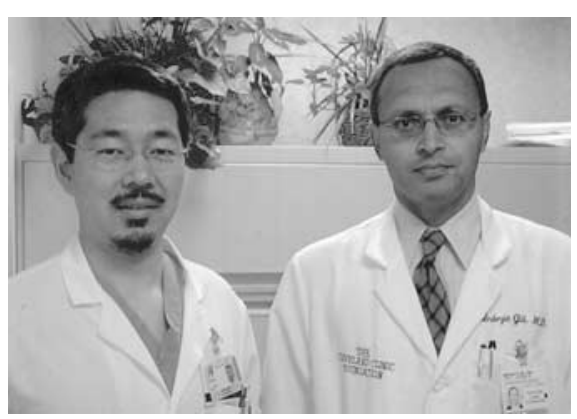

Izutani (left) and Gill (right)
A $\mathrm{n}$ intracoronary shunt is often used to maintain distal perfusion during off-pump coronary artery bypass grafting. We report the case of a patient who had acute graft occlusion caused by such a shunt as a result of intimal injury to the coronary artery during minimally invasive direct coronary artery bypass grafting (MIDCAB).

\section{Clinical Summary}

The patient was an 84-year-old woman who underwent cardiac catheterization after having abnormal results of a stress test before planned orthopedic surgery. Cardiac catheterization revealed triple-vessel coronary artery disease with $50 \%$ to $60 \%$ lesion of the left main trunk, mild and diffuse disease of the left anterior descending (LAD) coronary artery, normal left ventricular wall motion, and an ejection fraction of $79 \%$. Treatment options were discussed, and the patient was referred for a hybrid procedure, an elective MIDCAB to be followed by percutaneous intervention to the right coronary and circumflex system.

The patient underwent MIDCAB through a left minithoracotomy. The operative technique has been previously discussed

From the Department of Thoracic and Cardiovascular Surgery, Cleveland Clinic Foundation, Cleveland, Ohio.

Received for publication May 13, 2002; accepted for publication June 13, 2002.

Address for reprints: Inderjit S. Gill, FRCS(C), Department of Thoracic and Cardiovascular Surgery, Cleveland Clinic Foundation, 2500 MetroHealth Dr, Third Floor Hamann Building, Cleveland, OH 44109-1998 (E-mail: gillis@ccf.org).

J Thorac Cardiovasc Surg 2003;125:723-4

Copyright $\odot 2003$ by The American Association for Thoracic Surgery

$0022-5223 / 2003 \$ 30.00+0$

doi: $10.1067 / \mathrm{mtc} .2003 .142$ elsewhere. ${ }^{1}$ The diameter of the left internal thoracic artery (LITA) was $1.5 \mathrm{~mm}$. Stabilization of the target vessel was achieved with the Genzyme Immobilizer (Genzyme Corporation, Cambridge, Mass). Coronary arteriotomy was performed, and a $1.5-\mathrm{mm}$ intracoronary shunt (Guidant Axius Coronary Shunt; Guidant, Cupertino, Calif) was placed with some difficulty (Figure 1). The anastomosis was carried out with 7-0 Prolene running suture (Ethicon, Inc, Somerville, NJ). After the last stitch was placed, the intracoronary shunt was carefully taken out and the suture was tied. Graft flow measurements were carried out with a Transonic Flow-QC meter (Transonic Systems, Inc, Ithaca, NY). Although the graft pulsation was good, the graft flow measurement showed a poor diastolic flow pattern and insufficient blood flow at $5 \mathrm{~mL} / \mathrm{min}$. The anastomosis was therefore taken down and examined. No reason was found for graft malfunction. A $1.5-\mathrm{mm}$ vessel probe was passed through distally and proximally from the arteriotomy. The anastomosis was then carefully redone in the same fashion. However, a repeated graft flow measurement was still unsatisfactory with poor graft flow. The patient was taken for urgent cardiac catheterization, and the angiogram showed total occlusion of the LITA graft at the mid portion and total occlusion of the mid LAD.

The patient was taken back to the operating room and underwent a median sternotomy and conventional cardioplegic arrest. The anastomosis was taken down again and examined. Fresh thrombus formation was found blocking the blood flow at the anastomotic site, and this was removed cautiously. It appeared to have occluded both the proximal LAD and the LITA graft. The LAD arteriotomy was extended proximally and distally. Intimal lacerations were seen $5 \mathrm{~mm}$ proximal and distal to the arteriotomy (Figure 2, $A$ ), which coincided with the bulbous ends of the intracoronary shunt (Figure 1). Although no abnormality was found in the LITA graft at this site, it was abandoned for regrafting. The LAD was divided, two separate 